\title{
Analytical Considerations in Nanoscale Flow Cytometry of Extracellular Vesicles to Achieve Data Linearity
}

\author{
Janice Gomes ${ }^{1,2, *}$ Fabrice Lucien ${ }^{1,3,4, *}$ Tyler T. Cooper ${ }^{5}$ Yohan Kim ${ }^{1,3,4} \quad$ Karla C. Williams ${ }^{3,4}$ \\ XinYang Liao ${ }^{4}$ Lauren Kaufman $^{6}$ Francois Lagugné-Labarthet ${ }^{6}$ Oliver Kenyon ${ }^{7}$ Justin Boysen ${ }^{8}$ \\ Neil E. Kay ${ }^{8}$ Christopher W. McIntyre ${ }^{1,2}$ Hon S. Leong ${ }^{1,3,4}$
}

\footnotetext{
${ }^{1}$ Department of Pathology and Laboratory Medicine, University of Western Ontario, London, Ontario, Canada

2 Kidney Clinical Research Unit, London Health Sciences Centre, London, Ontario, Canada

${ }^{3}$ Department of Urology, Mayo Clinic, Rochester, Minnesota, United States

${ }^{4}$ Translational Prostate Cancer Research Laboratory, Lawson Health Research Institute, London, Ontario, Canada

${ }^{5}$ Department of Physiology and Pharmacology, University of Western Ontario, London, Ontario, Canada

${ }^{6}$ Department of Chemistry, University of Western Ontario, London, Ontario, Canada

${ }^{7}$ Apogee Flow Systems Inc., Hertfordshire, United Kingdom

8 Division of Hematology, Mayo Clinic, Rochester, Minnesota, United States
}

Thromb Haemost 2018;118:1612-1624.

Address for correspondence Hon S. Leong, PhD, Department of Urology, Mayo Clinic, 9-01B, Guggenheim Building, 200 First Street SW, Rochester, MN 55905, United States (e-mail: leong.hon@mayo.edu).

\begin{abstract}
Background Platelet microparticles (PMPs) and their abundance in the blood are a prognostic biomarker in thrombotic disorders and cancer. Nanoscale flow cytometry $(\mathrm{nFC})$ is ideal for high-throughput analysis of PMPs but these clinical assays have not been developed previously.

Objective This article demonstrates that $\mathrm{nFC}$ is a suitable technology to enumerate PMPs present in plasma samples in a clinical setting.

Materials and Methods nFC was performed using the Apogee A50-Micro instrument. Instrument settings and acquisition parameters were developed with the use of fluorescent beads and plasma samples. Sample preparation and handling was also optimized.

Results nFC allows for linear detection of particles between approximately 200 and $1,000 \mathrm{~nm}$ based on calibration beads and was dependent on dilution factor and flow rate. Linearity in event analysis as samples became more diluted was lost when events

Keywords

- cancer

- microparticles

- flow cytometry approximately $100 \mathrm{~nm}$ were gated while linearity was maintained despite dilution of sample in events larger than $200 \mathrm{~nm}$ in diameter. Higher flow rates lead to an underestimation of events analysed per microlitre of analyte and this was more pronounced when plasma samples were not diluted more than $1 / 20 \times$.
\end{abstract}

* Janice Gomes and Fabrice Lucien equally contributed to the study.

received

April 25, 2018

accepted after revision

July 11, 2018 (c) 2018 Georg Thieme Verlag KG Stuttgart · New York
DOI https://doi.org/

$10.1055 / \mathrm{s}-0038-1668544$. ISSN 0340-6245. 
Conclusion nFC offers multi-parametric analysis of PMPs when optimal calibration of acquisition and sample processing settings is performed. Analysis of plasmas from metastatic prostate cancer patients and leukaemia patients revealed that PMP levels were larger than $100 \mathrm{~nm}$ and were equally abundant in patients that responded to or failed androgen deprivation therapy or between patients representing different stages of leukaemia.

\section{Introduction}

Extracellular vesicles (EVs) such as microparticles (MPs) are cell-derived membrane vesicles that are released into bodily fluids following cell activation or apoptosis. ${ }^{1-3}$ Their budding from the cell is propagated by cytoskeletal re-arrangement and membrane asymmetry in a process called ectocytosis (membrane budding). ${ }^{4}$ MPs, also known as microvesicles and ectosomes, can be distinguished from other EVs such as exosomes and apoptotic bodies by their size (100-1,000 nm) and retention of surface antigens specific to donor cells., Originally, MPs were thought to be 'cell garbage' or 'platelet dust'. 7,8 However, these sub-micron particles have gained more interest as an important inter-cellular communication mediator involved in normal and pathological processes such as Alzheimer's disease, cancer and infectious diseases. ${ }^{9-11}$ Like the cells that released them, MPs are composed of nucleic acids, lipids and proteins, which in some cases, makes them an abundant source of biomarkers with potential functional impact.

Platelet microparticles (PMPs) are distinct from other MPs because they are a significant fraction of MPs in the blood of healthy individuals. ${ }^{12}$ Rises in PMP levels have been associated with various pathological conditions such as atherosclerosis, rheumatoid arthritis, thrombosis, inflammation and cancer. ${ }^{13-17}$ Therefore, PMP levels could contribute to multivariable analyses for non-invasive liquid biopsies to guide clinicians in pathological classification, diagnoses and potential treatments. While PMPs have the potential to be used in the clinical setting, methods of isolation, characterization and enumeration remain contentious as there is a lack of gold standard procedure(s). A rigorous standardization in PMP enumeration is required to assess their potential as a biomarker in disease.

Currently, microscopy, dynamic light scattering, nanoparticle tracking analysis (NTA), tunable resistive pulse sensing and flow cytometry (FC) are some physical methods that have been used to characterize MPs by cellular origin, size, population, number and structure. ${ }^{18-23}$ Of these methods, FC is advantageous because it has the ability to analyse individual EVs and detect two or more antigens simultaneously on their surface through the use of fluorophoreconjugated antibodies in a high-throughput and multiplexed manner. ${ }^{23}$ While conventional FC allows for rapid detection of antigen-specific MPs and size, it has limited sensitivity and can only detect particles as small as $300 \mathrm{~nm}$ based on light scattering. ${ }^{24}$ This limitation in MP detection may result in the under-estimation of PMP levels. ${ }^{4}$ More recently, few groups have studied MPs by using nanoscale flow cytometry (nFC) ${ }^{25-28}$ Although nFC is similar to conventional FC in terms of light scattering and high-throughput quantification, nFC is able to detect MPs as small as $100 \mathrm{~nm}$ with multiplex fluorescent detection. ${ }^{29-31}$ While the use of nFC is emerging as a suitable tool to enumerate MPs in a clinical setting, it still lacks analysis protocols that are supported by reproducibility and cross-validation studies. Furthermore, there is a need for standardization in terms of sizing parameters with the use of size-calibrated fluorescent beads, intra-instrument and inter-instrument reproducibility, detection of massive coincidence (swarm effect) and whole blood and plasma preparation and storage. $22,24,32,33$ While these are common areas of regulation among users of conventional FC, this level of standardization is also needed for FC instruments that analyse within the nanoscale realm (100-1000 nm). Here, we tested different conditions of plasma preparation and storage to optimize analysis of PMPs. Additionally, we developed a detailed methodology to accurately quantify sub-populations of PMPs from patient plasmas for future use in a clinical setting.

\section{Materials and Methods}

\section{Nanoscale Flow Cytometry Settings}

All samples were analysed using the A50-Micro Plus Nanoscale Flow Cytometer (Apogee FlowSystems Inc.) equipped with $70 \mathrm{~mW} 405 \mathrm{~nm}$ (violet), $53 \mathrm{~mW} 488 \mathrm{~nm}$ (blue) and $73 \mathrm{~mW} 639 \mathrm{~nm}$ (red) lasers. Parameters in the control panel were set to sheath pressure of 150 mbar and number of flush cycles to 3 . Sample flow rate of $1.5 \mu \mathrm{L} / \mathrm{min}$ (or as indicated) was used for all measurements (or as indicated) and the time of acquisition was held constant for all samples at 60 seconds to yield enough events. An illumination wavelength of $405 \mathrm{~nm}$ (70 mW) was used to detect scattered light by MPs. Before sample analysis, calibration of flow cytometer was performed using a reference bead mix (ApogeeMix, Apogee Flow Systems Inc.) composed of a mixture of silica nanoparticles with diameters of 180 , $240,300,590,880$ and $1,300 \mathrm{~nm}$ with a refractive index (RI) of 1.42; and 110 and $500 \mathrm{~nm}$ green fluorescent (excited by blue laser) polystyrene nanoparticles with an RI of 1.59 (latex) were used. These beads were used to assess the FC's light scattering detection of MPs and fluorescence detection resolution. Light scatter triggering thresholds were determined with the smallest particles distinguishable from noise (110 nm polystyrene beads). Thresholds were set at 20 a.u. (small angle light scatter [SALS]) and 25 a.u. (large angle light scatter [LALS]) to eliminate optical and 
electronic background noise without losing particles of interest. Photomultiplier tube (PMT) voltages were set as follows: LALS (320 V), SALS (300 V) and L488-Grn (425 V). All measurements were performed in log mode. Noise levels in PMT panel were kept below 0.6. Fluorescent latex nanoparticles (Tetraspek nanoparticles, Thermo Fisher Scientific) of 200 and 1,000 nm sizes were used in experiments to validate the effect of mass coincidence and verify accurate enumeration. Massive coincidence ('swarm effect') was tested by analysing various dilutions of plasma samples that were run at a slow $(1.5 \mu \mathrm{L} / \mathrm{min})$, moderate $(6 \mu \mathrm{L} / \mathrm{min})$ and fast $(10.5 \mu \mathrm{L} / \mathrm{min})$ rates. All dilutions were also enumerated by confocal microscopy (Nikon Fast A1R,$+ 60 \times$ objective) and counted using the Fiji software. nFC performance was also compared with a conventional flow cytometer BD FACS Canto (BD Biosciences Inc.) by using $880 \mathrm{~nm}$ silica beads and 1,000 nm latex beads.

\section{Nanoscale Flow Cytometer Fluorescence Sensitivity}

Fluorescence calibration was performed using commercial spectral matching fluorescein isothiocyanate (FITC) fluorescent particle beads (Cat No. ECFP-F1-5K, lot AJ01, Spherotech Inc.) in which the fluorescence intensity had been calibrated in units of mean equivalent soluble fluorochromes (MESFs). Standard curve was created to assign MESF values to the fluorescent bead mix under the same instrument settings used for experiments. A 488-nm (50 mW) laser with a 530/40 filter was used to detect FITC fluorescence. Flow rate was set at $1.5 \mu \mathrm{L} / \mathrm{min}$ and PMT set at $475 \mathrm{~V}$.

\section{Plasma Samples}

Blood was collected from three healthy donors (25, 32 and 45 years of age) using a 21-gauge needle. The first $3 \mathrm{~mL}$ of blood was discarded and approximately $8 \mathrm{~mL}$ was transferred within sodium citrate Vacutainers (BD Biosciences Inc.). Platelet-free plasma (PFP) was isolated within 1 hour post-collection by double centrifugation at $2,500 \times g$ for 15 minutes (room temperature [RT], no brake) according to the International Society on Thrombosis and Haemostasis (ISTH) guidelines. ${ }^{34}$ Plasmas were aliquoted and stored at $-80^{\circ} \mathrm{C}$. Chronic lymphocytic leukaemia (CLL) plasma samples consisting of both low radioactive iodine (RAI) risk (stage 0-1) and high RAI risk (stage 3-4) were obtained from the Division of Hematology (Dr. Neil Kay) under Institutional Review Board \#1827. PFPs were prepared as above and stored at $-80^{\circ} \mathrm{C}$ until further use.

\section{Prostate Cancer Plasma Samples}

Blood was collected from 29 metastatic prostate cancer (mPCa) patients (stage IV) obtained through the Ontario Institute for Cancer Research Tumour Bank and the University Health Network Genitourinary BioBank (Toronto, Ontario, Canada) with Western University Research Ethics Board-approved Ethics Applications \#103156 and 103409 (-Table 1). Whole blood was collected within K2-EDTA Vacutainers (BD Biosciences Inc.). Blood was centrifuged at $1,500 \times g$ for 10 minutes, and the plasma supernatant was then reserved, aliquoted and stored at $-80^{\circ} \mathrm{C}$.
Table 1 Key clinical features of prostate cancer patients in -Fig. 7

\begin{tabular}{|l|l|}
\hline Clinical feature & Number of patients (\%) \\
\hline Median age (range) & $68.3(51.6-81.6)$ \\
\hline PSA level (ng/mL) & \\
\hline$<4$ & $6(21)$ \\
\hline $4-10$ & $3(10)$ \\
\hline$>10$ & $20(69)$ \\
\hline
\end{tabular}

Abbreviation: PSA, prostate-specific antigen.

\section{Variation of Handling and Processing of Whole Blood} Various storage experiments were conducted to analyse MP integrity with varying temperature, length of storage and thawing conditions. PFP was stored at RT, $4^{\circ} \mathrm{C},-20^{\circ} \mathrm{C}$ and $-80^{\circ} \mathrm{C}$ and was analysed in terms of total MPs and PMPs every week for 4 weeks. Additionally, PFP aliquots of $-80^{\circ} \mathrm{C}$ plasma samples were analysed following six freeze/thaw cycles with thawing at different temperatures (ice, $4^{\circ} \mathrm{C}$, RT and $37^{\circ} \mathrm{C}$ ).

\section{Immunostaining of Platelet Microparticles}

Titration of all detecting antibodies was performed with preconjugated clones and dilutions were determined from the original concentration $(\mu \mathrm{g} / \mathrm{mL})$ as provided by the manufacturer. Dilutions of Tetraspek nanoparticles/beads $(5,000 \times$, $10,000 \times, 20,000 \times, 40,000 \times$ and $80,000 \times)$ were performed using $0.20 \mu \mathrm{m}$ filtered phosphate-buffered saline (PBS). Dilutions of pre-conjugated antibodies (0.0125, 0.0250, $0.050, \quad 0.100 \mu \mathrm{g} /$ reaction) were also performed using $0.20 \mu \mathrm{m}$ filtered PBS. To enumerate PMPs in each of the experiments, $10 \mu \mathrm{L}$ of plasma was taken and incubated with 25 ng of CD41a-FITC (HIP8 clone, BD Biosciences Inc.) for 30 minutes at RT in the dark. Samples were also incubated with isotype-matched antibody IgG1k-FITC (MOPC-31C clone, BD Biosciences Inc.) to determine the non-specific binding and autofluorescence within each sample. The sample was then further diluted with PBS and run using the nFC. All experiments were done in triplicate. In experiments where Triton X-100 was added to patient plasma, $10 \mu \mathrm{L}$ of plasma stained with CD41a-FITC antibody was incubated with $0.5 \%$ of Triton X-100 for 30 seconds prior to analysis.

\section{Confocal Microscopy of Calibration Beads}

A Fast A1R confocal microscope equipped with 405/491/565/ $643 \mathrm{~nm}$ solid state lasers was used to perform imaging of calibration beads. In summary, $0.5 \mu \mathrm{L}$ of each diluted calibration bead mixture was placed onto a slide and coverslip. A $60 \times$ oil immersion objective lens was used to visualize the beads and the entire coverslip was imaged by using the NIS Elements software.

\section{Statistical Analysis}

Parameters recorded were as follows: total MPs within

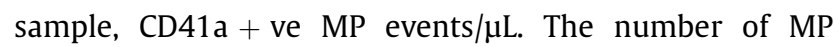
events/ $\mu \mathrm{L}$ in the isotype sample was subtracted from the MP events/ $\mu \mathrm{L}$ in the antibody sample. GraphPad Prism 7.0 
was used to run statistical analysis. Two-way analysis of variance test was used to evaluate statistical significance across the groups. The confidence interval was set at $95 \%$ and a $p$-value of $<0.05$ was considered significant.

\section{Results}

\section{Linear Light Scatter Detection of Nanoscale Events Regardless of Dilution Factor}

The A50-Micro Plus nFC relies on any three parameter-based triggers to analyse EVs: SALS, LALS and/or the fluorescence channel(s) of interest. Sensitivity of the A50-Micro Plus for sub-micron particles is based on the incident wavelength used to detect scattered light. The A50-Micro Plus uses a 405-nm laser instead of a 488-nm laser present on conventional flow cytometers. $^{35,36}$ In - Fig. 1A, a mix of fluorescent and nonfluorescent calibration beads was used to create various gates corresponding to different calibration size beads, and when presented on the LALS versus SALS plot provides a sizing gradient for each bead (- Fig. 1A). The fluorescent 110 and $500 \mathrm{~nm}$ beads are visibly distinct sub-populations (FL488, -Fig. 1A, middle plot) indicating that there is negligible 'swarm effect', that is, other FL488 events that are not 110 or $500 \mathrm{~nm}$ in diameter. The triggering threshold for LALS and SALS was optimized to maximize sensitivity of all true EVs while minimizing dark current noise (LALS 25 a.u, SALS 20 a. $\mathrm{u}$ ). Calibration beads from known concentrations were enumerated and for every single size particle count did not differ significantly with a coefficient of variation below $10 \%$ (-Table 2). Serial dilutions of $180 \mathrm{~nm}$ (RI, 1.42) did not produce a linear correlation between dilution factor and event levels beyond a dilution factor of $1 / 4 \times$ ( - Fig. 1B, left panel). Note that $880 \mathrm{~nm}$ silica beads produced a linear correlation between dilution factor and event levels (-Fig. 1B, right panel). Hence, analytical performance of the A50-Micro is affected by smaller particles $\left(r^{2}=0.9512\right)$ that are close to the lower detection limit of the instrument. In contrast, light scatter detection of $880 \mathrm{~nm}$ silica beads was not achievable with a conventional flow cytometer (BD FACS Canto) (-Supplementary Fig. S1, available in the online version).

Latex beads are also used for calibration and to assess light scatter performance of flow cytometers albeit having a higher RI (1.59) than silica beads and biological vesicles. Serial dilutions of latex bead (200 and $1000 \mathrm{~nm}$ ) concentrations were found to generate linear correlations between dilution and event rates (-Fig. 1C). Additionally, the MP count of beads that was determined with nFC was confirmed via confocal microscopy of the various diluted bead preparations ( - Fig. 1D, E). Serial twofold dilutions of plasmas from three healthy donors reveal linear relationships and demonstrates that 'true' events are recorded and enumeration is predominantly caused by single vesicles and not by massive coincidence when using light scatter detection ( - Fig. 1D, E, -Supplementary Fig. S1, available in the online version). ${ }^{24}$

\section{Impact of Flow Rate on EV Enumeration by nFC}

Flow rate in cytometry is a critical parameter for linear detection and concentration measurements of MPs. ${ }^{37}$ An excessively high flow rate may result in swarm effect when multiple small vesicles below the detection limit appear as a single event during acquisition. A lower flow rate provides suitable separation between events during acquisition, thereby increasing reproducibility of measurements. ${ }^{37}$ The Apogee A50-Micro Plus allows for acquisition at flow rates as low as $0.75 \mu \mathrm{L} / \mathrm{min}$ because it is equipped with a Hamilton syringe-driven delivery system to control flow rate. Serial dilutions of $180 \mathrm{~nm}$ silica beads were analysed at three different flow rates (1.5, 6.01 and $10.5 \mu \mathrm{L} / \mathrm{min}$ ) (-Fig. 2A) with no differences observed in event rates compared with theoretical bead concentrations. However, analysis of patient plasma samples did not consistently generate linear correlations when dilution was varied (-Fig. 2B). Two patient samples (\#1 and \#3) revealed discrepancy in particle number at lower dilutions, likely due to the larger proportion of events $<180 \mathrm{~nm}(5 \times$ and $2 \times$ more events in the $180-\mathrm{nm}$ gate in plasmas \#1 and \#3) in those two plasma samples (-Fig. 3). These patient samples also exhibited a higher concentration of total MPs compared with sample \#2. At higher flow rates, particle number exceeds $\mathrm{nFC}$ acquisition limit resulting in an under-estimation of MP concentrations. At $10.5 \mu \mathrm{L} / \mathrm{min}$, the number of events per second recorded was 38,640 (plasma \#1), 11,585 (plasma \#2) and 16,218 (plasma \#3) at dilution of $1 / 20 \times$. The Apogee A50-Micro Plus is only reliable for light scatter-based enumeration of MPs when the event rate does not exceed approximately 12,000 events per second. This suggests that in addition to dilution factor, flow rate is also critical for linear and accurate enumeration of MPs.

\section{Detection of Platelet Microparticles by nFC from Human Plasmas}

Accurate and reproducible enumeration of MP or EV populations of interest requires detectors with high sensitivity on both light scatter and fluorescence detection. Most conventional flow cytometers used for MP analysis prioritize fluorescence sensitivity over scatter detection performance suggesting that fluorescence-based triggering is a more accepted alternative to detect fluorescent-labelled MP sub-sets. ${ }^{38,39}$ A recent study indicated that fluorescence resolution limit of the A50-Micro Plus is 304 MESF-FITC which is similar to high-sensitive flow cytometers. ${ }^{36,39}$ We assessed the A50-Micro Plus performance to enumerate CD41a-positive PMPs using scatter and fluorescence triggering. Fluorescence calibration of the A50-Micro Plus was performed by using FITC polystyrene beads of known intensities ( - Fig. 4A, B). The smallest particles distinguishable over the noise (110 $\mathrm{nm}$ polystyrene beads) revealed a value of 12,500 MESF-FITC. Mean fluorescence intensities produced by CD41a-positive PMPs in human PFP do not change regardless of dilution which confirms the lack of massive coincidence during PMP enumeration by $\mathrm{nFC}$ (-Table 3). PMPs were quantified from human PFP by using immunolabelling with an antibody against CD41a. ${ }^{40}$ Antibody titration curves were generated to identify the appropriate antibody concentration for immunostaining (-Fig. 4C, D). This population was abolished when Triton 
A

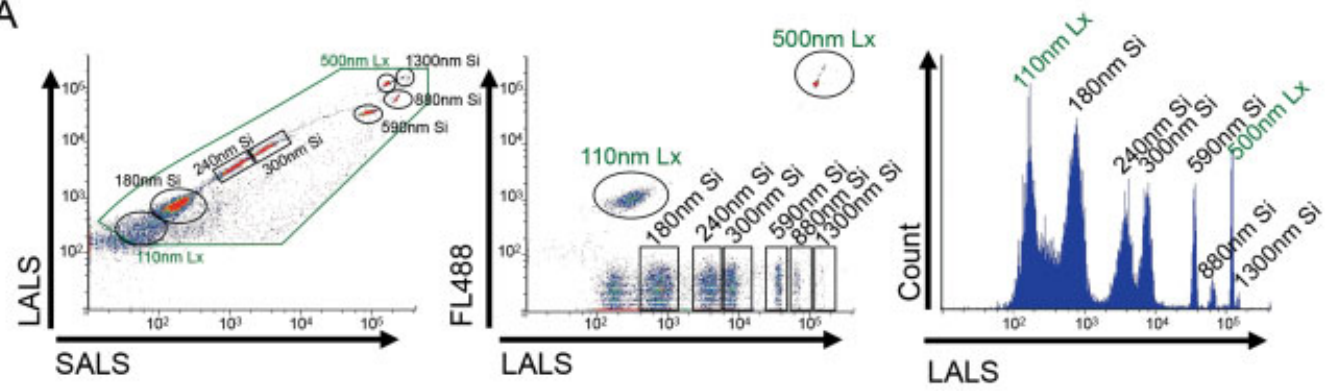

B
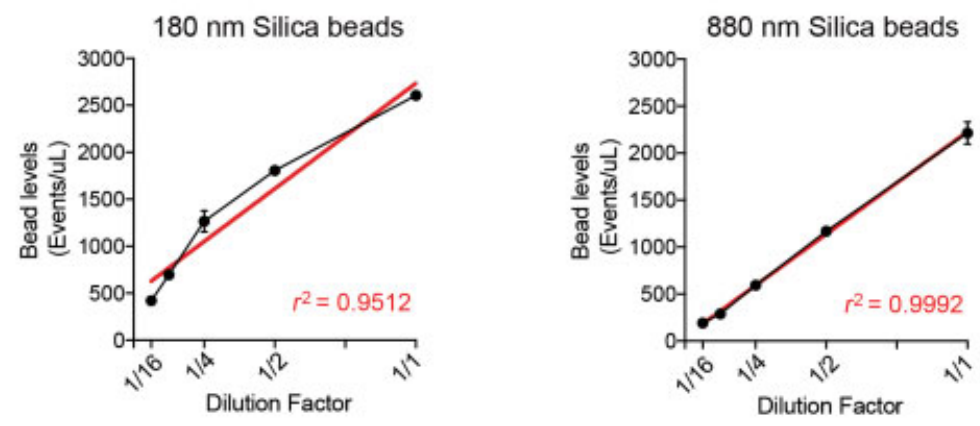

C

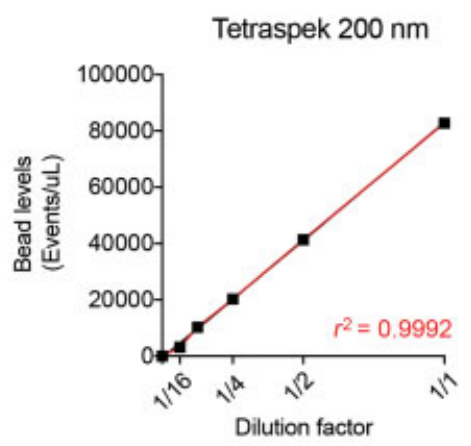

D
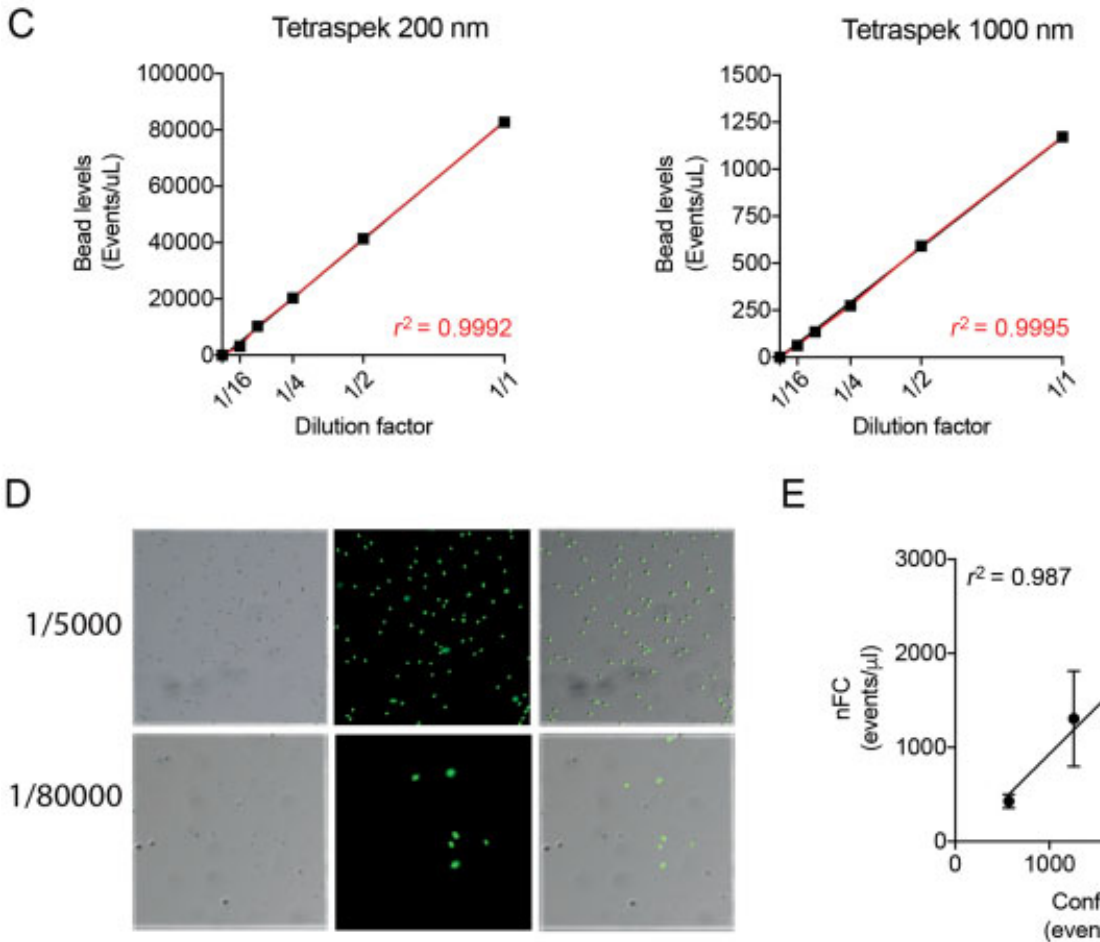

E

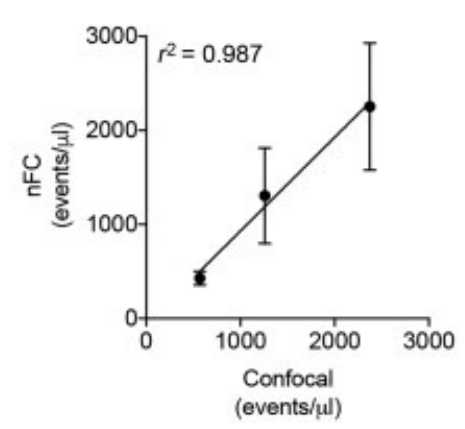

Fig. 1 Linearized detection of microparticles by using calibration sized beads and nanoscale flow cytometry and confocal microscopy. (A) Calibration of the flow cytometer with a bead mix of fluorescent latex and non-fluorescent silica beads. Cytograms and histograms show bead populations detected with light-scatter and fluorescence. The green box indicates the sort gate for microparticle-related events based on lightscatter detection of calibration beads. (B) Enumeration of 110 and $880 \mathrm{~nm}$ silica beads at various serial dilutions. (C) Enumeration of 0.2 and $1 \mu \mathrm{m}$ Tetraspek bead dilutions. Linear regression was performed on serial dilution graphs representing technical triplicates. (D) Representative confocal microscopy images of the fluorescent $1 \mu \mathrm{m}$ latex beads deposited onto a haemocytometer. Lower panel are images presented at higher power. (E) Correlation curve of bead counts with nanoscale flow cytometer and confocal microscope. Each experiment represents three technical replicates.

$\mathrm{X}$-100 was added to permeabilize PMPs, resulting in more events with lower LALS and SALS being abundant. This observation is consistent with previous reports of Triton $\mathrm{X}-100$ inducing lysis of microvesicles leading to smaller events being generated. ${ }^{41}$ Interestingly, the number of CD41a-positive MPs (PMPs) when detecting with a light scatter trigger or a fluorescence trigger were similar in three different healthy volunteer PFP samples (-Fig. 4E). 
Table 2 Accurate enumeration of calibration beads based on light scatter threshold

\begin{tabular}{|l|l|l|l|l|}
\hline Size & Count $(\mathrm{EV} / \mu \mathrm{L})$ & Fluorescent & Counted & $\% \mathrm{CV}$ \\
\hline 110 & 8,000 & Green & 8,386 & 4.80 \\
\hline 180 & 23,000 & None & 21,749 & 5.40 \\
\hline 240 & 10,000 & None & 10,732 & 7.30 \\
\hline 300 & 9,000 & None & 9,304 & 3.30 \\
\hline 500 & 3,600 & Green & 3,794 & 5.30 \\
\hline 590 & 2,700 & None & 2,767 & 2.40 \\
\hline 880 & 3,900 & None & 4,214 & 8 \\
\hline 1,300 & 3,400 & None & 3,122 & 9.80 \\
\hline
\end{tabular}

Abbreviations: CV, coefficient of variation; EV, extracellular vesicle.

PFP stored at different temperatures $\left(\mathrm{RT}, 4^{\circ} \mathrm{C},-20^{\circ} \mathrm{C},-80^{\circ} \mathrm{C}\right)$ for up to 4 weeks was analysed to determine total MP counts and CD41a + ve MPs levels. Storage at RT induced a significant decrease of total MPs after a 1 -week period (- Fig. 5A), whereas other storage conditions did not affect total MP count ( - Fig. 5B-D). Storage at $-80^{\circ} \mathrm{C}$ led to the lowest amount of PMP loss regardless of storage timeframe ( - Fig. 5D). Storage at RT or $4^{\circ} \mathrm{C}$ led to a complete loss of PMPs after 1 week and a partial loss of approximately $40 \%$ of PMPs was observed at $-20^{\circ} \mathrm{C}$ after 4 weeks (-Fig. 5A-C).

To assess how MP integrity is affected by thawing of plasma, we enumerated total MPs, PMPs from platelet-poor plasma following multiple freeze/thaw cycles ( - Fig. 6). PMP levels did not change after repeated freeze thaws, regardless of thawing conditions $\left(37^{\circ} \mathrm{C}, \mathrm{RT}, 4^{\circ} \mathrm{C}\right.$, on ice).

\section{Detection of PMPs in Metastatic Prostate Cancer Patient Plasmas}

Cancer progression is often accompanied by platelet activation $^{42}$ and the latter is known to be associated with MP release. We hypothesized that PMP release can be a predictor of prostate cancer responsiveness to docetaxel treatment. Twenty-nine plasmas from patients with mPCas treated with docetaxel were analysed for PMP levels (-Table 1) (-Fig. 7A, B). PMP levels were not different between patients who responded to docetaxel versus those resistant to treatment.

\section{Enumeration of PMPs in CLL Patient Plasmas}

Patients with high RAI risk of CLL are characterized by an increasing leukaemia burden, often with increase in absolute blood leukaemic cells, and a significant decrease of platelet counts (i.e. detectable thrombocytopaenia). Since PMPs are generated by platelet activation, levels of PMPs vary between patients with low RAI risk and patients with high RAI risk were enumerated via nFC. Interestingly, we observed that levels of PMPs were slightly lower in patients with high RAI compared with patients with low RAI ( - Fig. 7C-E), but this did not reach statistical significance. The average number of PMPs was $164,827 \pm 24,885$ PMPs per microlitre of plasma and $114,938 \pm 24,404$ PMPs per microlitre of plasma in low and high RAI patients, respectively (mean \pm standard error, -Fig. 7D). Relative abundance of PMPs in patient plasmas

A

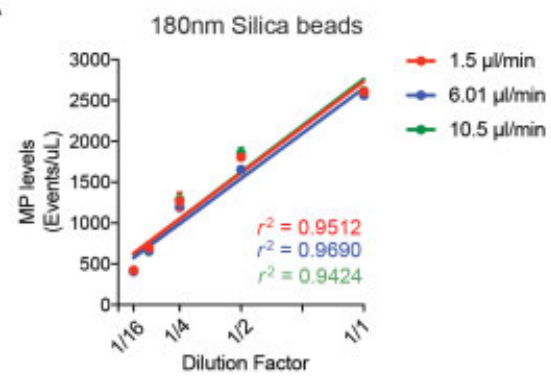

B
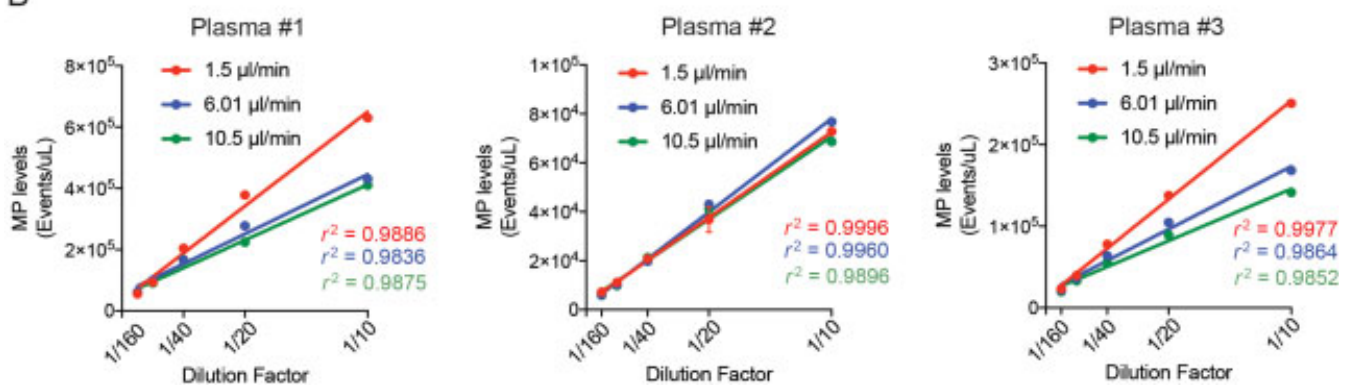

Fig. 2 Impact of flow rate on extracellular vesicle (EV) enumeration by nanoscale flow cytometry. Serial dilutions of $180 \mathrm{~nm}$ silica beads (A) and three human plasmas (B) were analysed at different flow rates: low $(1.5 \mu \mathrm{L} /$ min), medium $(6.0 \mu \mathrm{L} / \mathrm{min})$ and high $(10.5 \mu \mathrm{L} / \mathrm{min})$. 

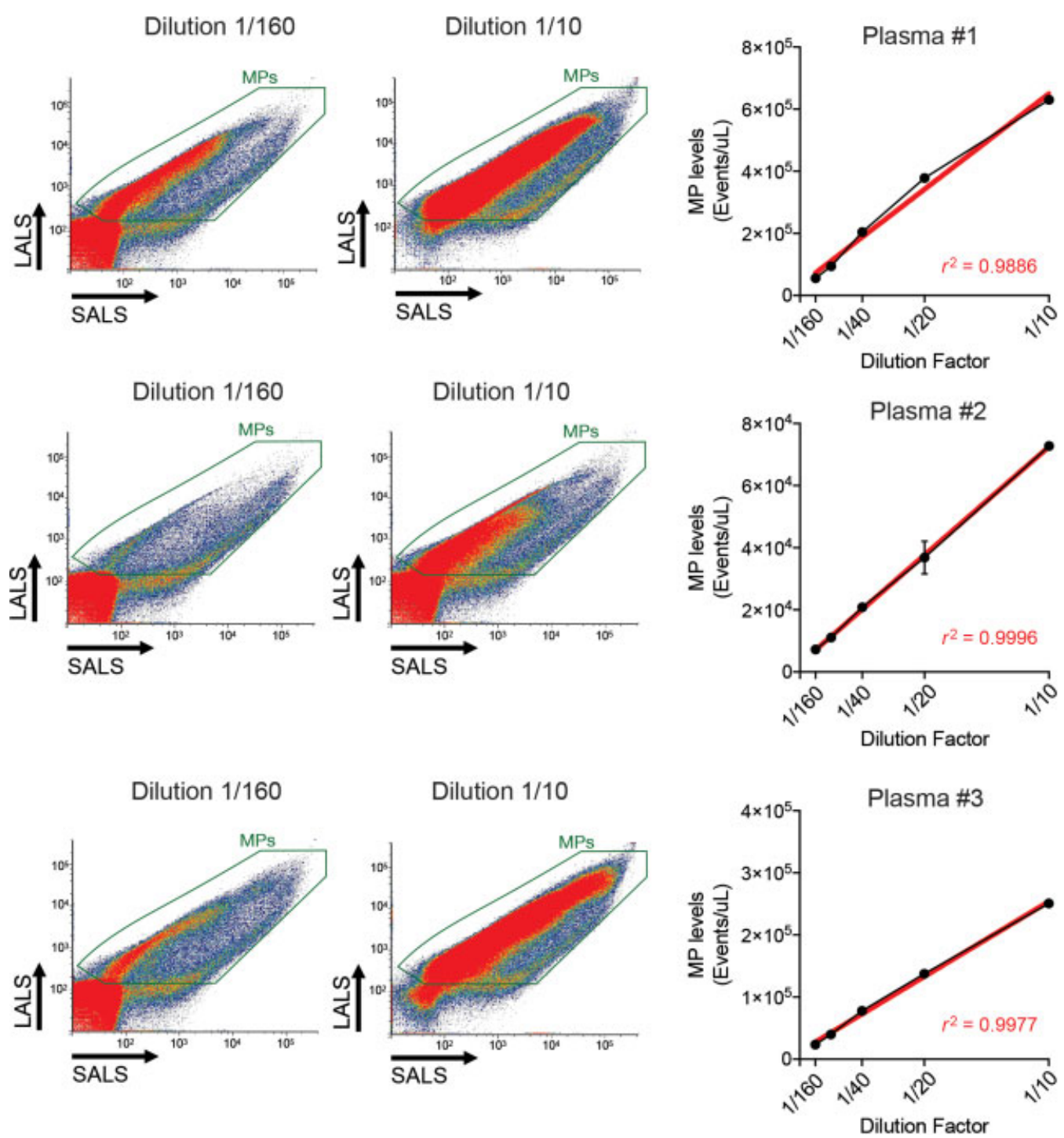

Fig. 3 Linearized detection of microparticles from healthy donor plasmas by nanoscale flow cytometry (nFC) and validation of $\mathrm{nFC}$ performance by confocal microscopy. Cytograms show light scatter microparticle (MP) detection (large angle light scatter/small angle light scatter [LALS/ SALS]) in plasmas of three healthy donors at two different dilutions. Graphs represent enumeration of MPs in plasma serial dilutions. Trend lines for each enumeration were recorded to confirm linearity of dilutions.

was also decreased in patient with high RAI risk of CLL (-Fig. 7E).

\section{Discussion}

$\mathrm{nFC}$ is a powerful means of performing multi-parametric analysis of EVs, carrying the same concepts of 'fluorescenceactivated cell sorting (FACS)' for cell immunophenotyping over to EVs. This has enabled investigators to use many cytometry compatible antibodies for use in this emerging area of EV analysis. Our main findings in this work are that the conditions for MP/EV analysis by nFC are highly dependent on flow rates and the dilution factors used when processing and preparing plasma samples for analysis. What was not previously appreciated was the fact that plasma contains a much higher concentration of EVs than previously thought and is at a level that could exceed analysis rates if not accounted for. For our study, we focused on PMPs because of their relative abundance and variation in certain disease phenotypes such as sepsis, ${ }^{43}$ thrombosis ${ }^{44}$ and cancer. ${ }^{45}$ The scientific committees for the ISTH have instituted several recommendations for standardization of PMPs enumeration by FC and inter-laboratory reproducibility. ${ }^{33,46}$ Overall, by using nanoscale or high-resolution FC instrumentation for EV research, we have confirmed findings from previous reports and offer additional recommendations for plasma processing and handling, as well as PMP enumeration that would make PMP analysis feasible in a clinical laboratory environment.

Blood collection and sample preparation are critical to maintaining PMP integrity and concentration. Sodium citrate appears to be the best anticoagulant during blood collection to minimize in vitro platelet activation and MP release induced by other anticoagulants such as ethylenediaminetetraacetic acid or heparin. Following blood draw, samples must be consistently processed in a short time and should undergo two centrifugations at 2,500 $\times \mathrm{g}$ for 15 minutes to 
A
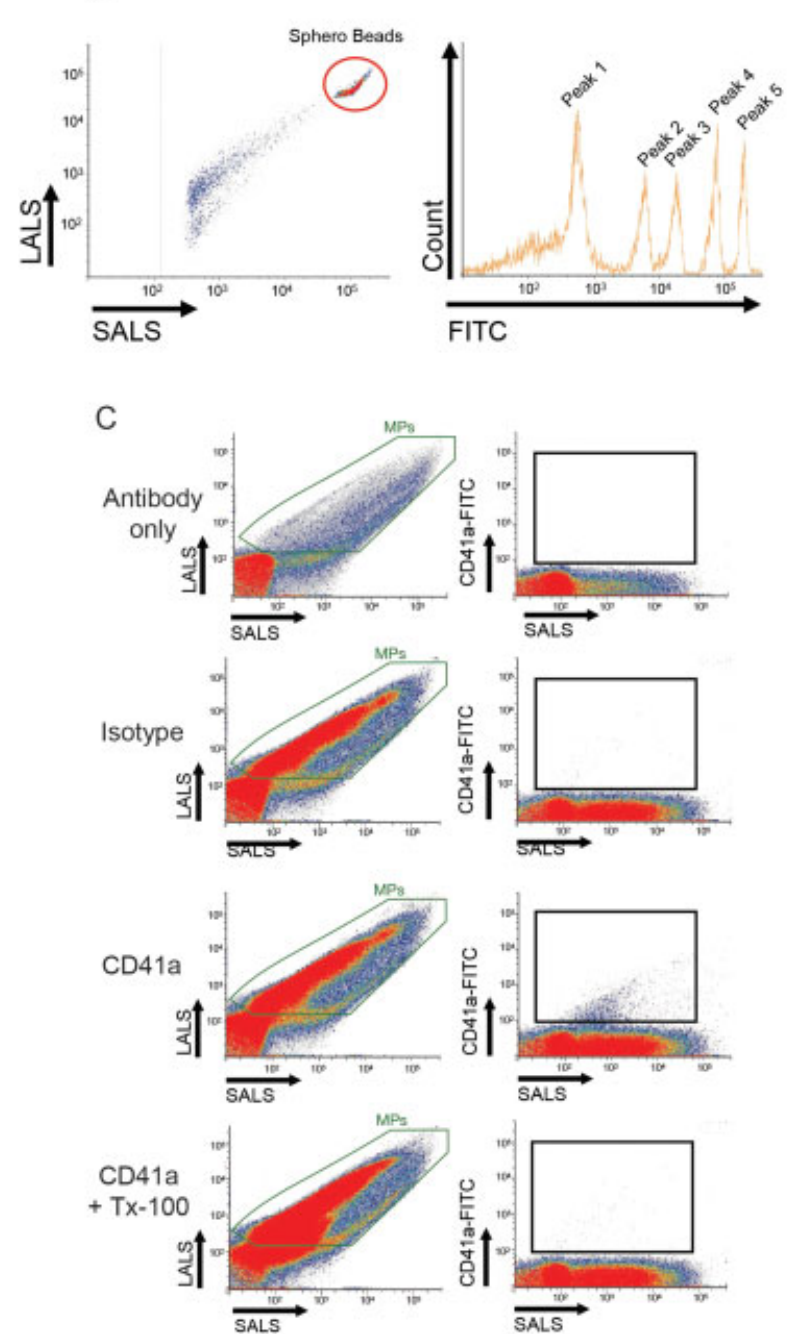

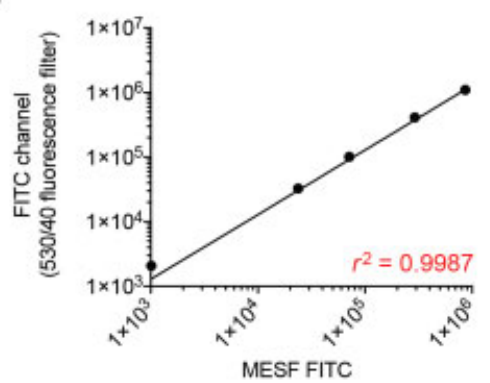

D
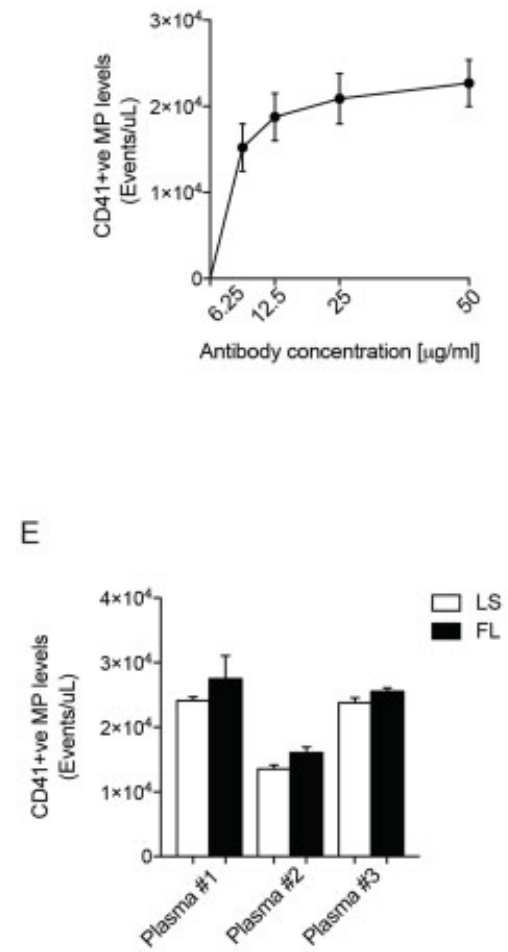

Fig. 4 Detection of platelet microparticles (PMPs) by nanoscale flow cytometry ( $\mathrm{nFC}$ ). (A) Representative cytograms showing Spherotech beads used for mean equivalent soluble fluorochrome (MESF) calibration. Five peaks of different MESF intensities were accordingly detected by nFC. (B) Linear regression of MESF versus green channel indicating intensities of every peak detected by the fluorescein isothiocyanate (FITC) detector. (C) CD41a-positive platelet microparticles in human plasmas. Note that $0.025 \mu \mathrm{g}$ of CD41a-FITC antibody or isotype-matched control were used. Plasma immunostained with anti-CD41a-FITC antibody and then treated with Triton X-100 (final concentration of $0.5 \%$ ) (bottom panel). (D) Antibody titration curve was performed with human platelet-free plasma. (E) CD41a-positive PMPs levels in three human plasmas analysed with light scatter triggering threshold or fluorescence threshold. Bars represent mean \pm standard error of the mean (SEM) for $n=3$ independent experiments.

Table 3 Mean fluorescence intensities of CD41a + ve PMPs does not change with plasma dilutions

\begin{tabular}{|l|l|l|l|}
\hline & Plasma \#1 & Plasma \#2 & Plasma \#3 \\
\hline Dilution $1 / 10$ & $2,056.1$ & 2,361 & 2,603 \\
\hline Dilution $1 / 20$ & $1,937.1$ & 2,497 & 2,578 \\
\hline Dilution $1 / 40$ & $2,045.9$ & 2,520 & 2,575 \\
\hline Dilution $1 / 80$ & $2,101.8$ & 2,579 & 2,659 \\
\hline Mean \pm SD & $2,583 \pm 89$ & $2,489 \pm 92$ & $2,603 \pm 39$ \\
\hline
\end{tabular}

Abbreviations: MESF, mean equivalent soluble fluorochrome; PMP, platelet microparticle; SD, standard deviation.

Note: Values are expressed in MESF units. obtain clinical quality PFP. In addition, cryopreservation has an effect on PMP levels and integrity. In all situations, $-80^{\circ} \mathrm{C}$ appears to be ideal for maintaining the original $\mathrm{EV}$ profile of the plasma sample. Repeated freeze/thaw cycles did not appear deleterious, although minimizing freeze/thaw cycles is still recommended. Interest for plasma analysis in MP/EV research is growing since large institutional biobanks store patient plasmas in anticipation for their use in retrospective biomarker-based studies. Unfortunately, blood collection and plasma isolation are not consistently controlled in such clinical studies. While prospective clinical studies are required to include the latest recommendations in terms of sample handling and processing, retrospective studies must 
A
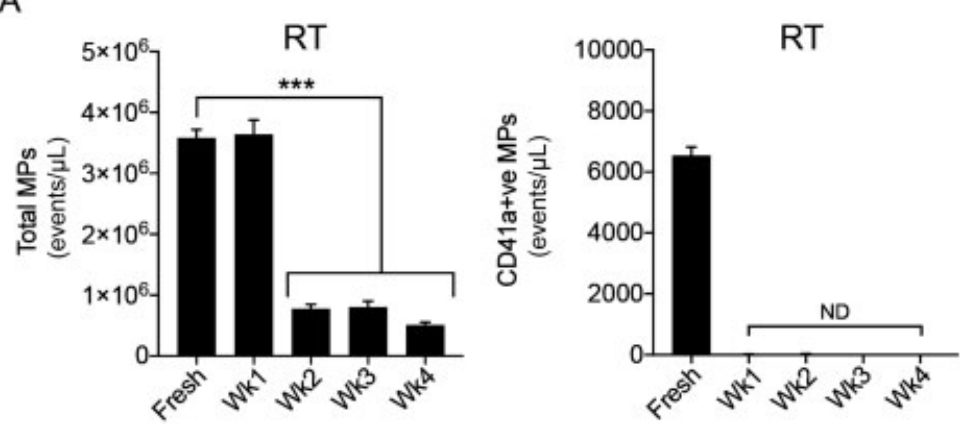

в
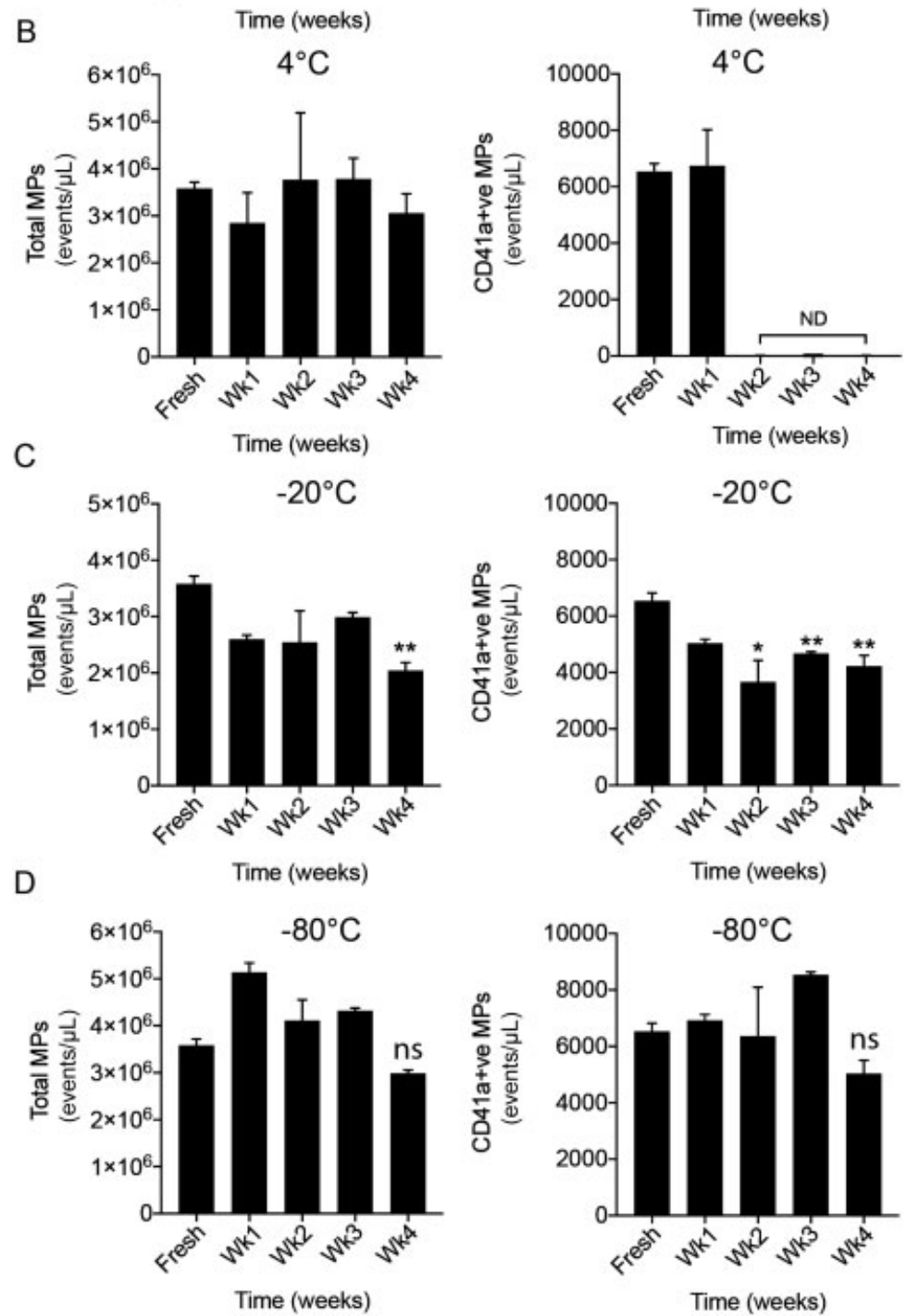

Fig. 5 Effect of storage of plasma samples over 4 weeks at various temperatures. (A) Enumeration of total microparticles (MPs) and CD41a + ve MPs from plasma stored for 4 weeks at room temperature (RT), (B) at $4{ }^{\circ} \mathrm{C},(\mathrm{C})$ at $-20^{\circ} \mathrm{C}$ and (D) at $-80^{\circ} \mathrm{C}$ for 4 weeks. Bars represent mean \pm standard error of the mean (SEM) for $n=3$ independent experiments. Two-way analysis of variance (ANOVA) test, ${ }^{*} p<0.05$, ${ }^{* *} p<0.01$ and ${ }^{* * *} p<0.001 ;$ ns $=$ not significant, nd = non-detectable.

also provide these details if similar studies are to be done and results to be compared.

The opportunities afforded by $\mathrm{nFC}$ are numerous; only 10 to $20 \mu \mathrm{L}$ of plasma sample is required for analysis which is not processed and is subsequently diluted 10 to $60 \times$ fold for final analysis. Moreover, 20 to $50 \mathrm{ng}$ of pre-conjugated antibody is needed for analysis at this scale, whereas approximately $1 \mu \mathrm{g}$ is often used to immunostain cells prior to 'FACS' analysis. Moreover, the antibody titration experiments show that there is no need to wash EVs even if feasible for the investigator because there is a lack of non-specific signal/events when antibody alone is analysed. This is an important benefit and avoids the need for time-consuming ultra-centrifugation steps. Exploiting the full capacity offered by $\mathrm{nFC}$ does require 


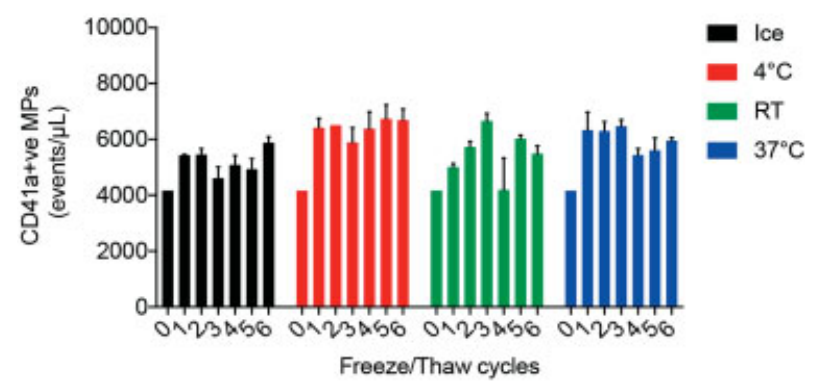

Fig. 6 Impact of freeze thaw cycles of human plasmas on microparticle (MP) integrity and enumeration. Enumeration of CD41a + ve MPs of a healthy control platelet-free plasma (PFP) sample that was frozen at $-80^{\circ} \mathrm{C}$ and differentially thawed gently on ice (black), at $4^{\circ} \mathrm{C}$ (red), at room temperature (RT) (green) and at $37^{\circ} \mathrm{C}$. Bars represent mean \pm standard error of the mean (SEM) for $n=3$ independent experiments.

many technical considerations to ensure accurate enumeration of MPs beyond optimal sample preparation and handling. For instance, antibody titration experiments along with isotypes are pre-requisite to determining the optimal antibody concentration to be used. On that note, antibody working concentration may differ depending on sample type (culture media, plasma, urine, and so on) where MP/EV concentrations are not identical in body fluids. Sample dilution must be tested to determine the full range of dilutions responding to linearity and to avoid saturation of acquisition capacity of nFC as well as too dilute of a concentration for each sample. Use of isotypematched controls is also required for gating and control of background to minimize inter-experimental batch-to-batch variability. Differences in non-specific binding present between plasma samples justify the inclusion of isotype controls. Interestingly, Andersen et al raise some concerns regarding the use of isotype-matched controls and recommend incubation of plasma samples with a blocking reagent prior to FC analysis. ${ }^{47}$ Therefore, it would be relevant to compare blocking reagent versus isotype control in terms of background levels for EV analysis by nFC.

EVs have demonstrated great potential as translational biomarkers in several disease conditions, but biomarker validation for future clinical use requires large number of samples to be analysed. The ideal technology for EV-based A
Patient
$\# 08246$
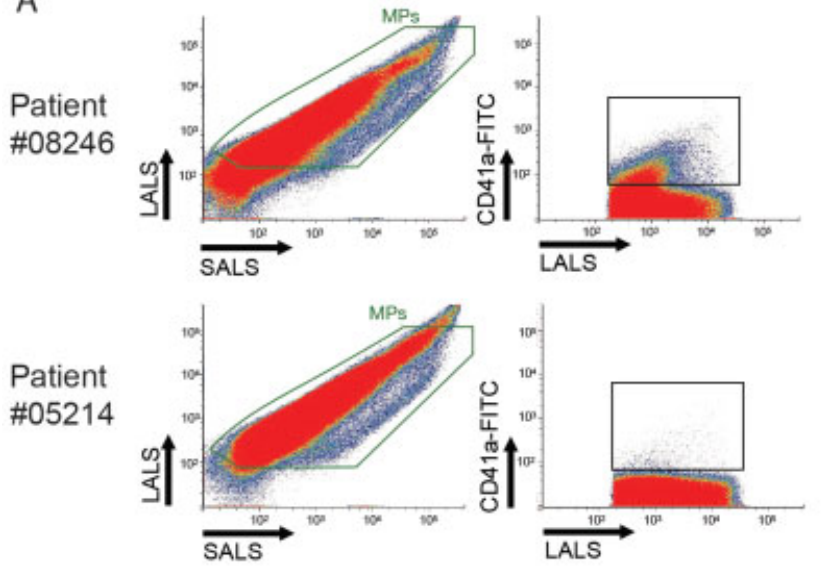

B

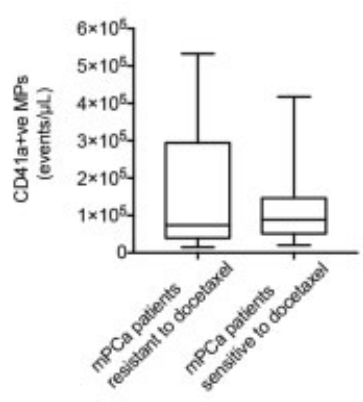

C
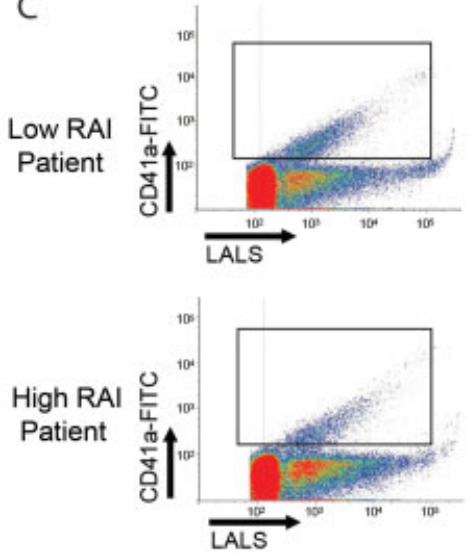

D

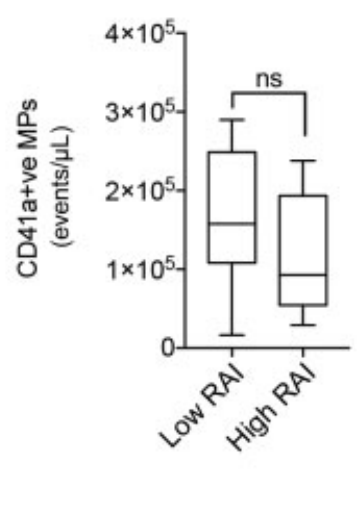

$\mathrm{E}$

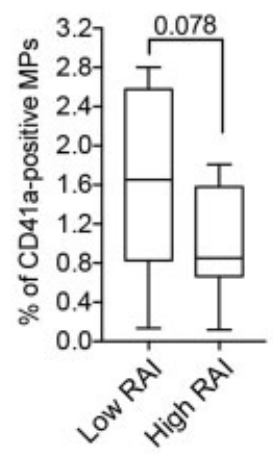

Fig.7 Quantification of platelet microparticles (PMPs) in metastatic prostate cancer patients treated with docetaxel patients with low and high radioactive iodine (RAI) risk of chronic lymphocytic leukaemia (CLL). (A) Representative cytograms of total microparticles (MPs) and CD41a + ve MPs in two patients expressing either low or high levels of PMPs. (B) Box plots showing PMP enumeration in metastatic prostate cancer patients sensitive $(n=12)$ or resistant $(n=17)$ to docetaxel treatment. (C) Representative cytograms of total MPs and CD41a + ve MPs in two patients with low or high risk of CLL. (D) Box plots showing PMP enumeration CLL patients low RAI $(n=11)$ or high RAI $(n=11)$. (E) Box plots showing percentage of PMPs in blood of CLL patients low RAl or high RAI. Bars represent mean \pm standard deviation (SD). Student's $t$-test, ns = non-significant. 
biomarker validation will allow for accurate particle enumeration, multi-parameter phenotyping in a high-throughput manner. Even though no such technology is commercially available, FC is the most suitable and fulfils these criteria. Advanced optical methods including NTA, electron microscopy, atomic force microscopy and highresolution microscopy have revolutionized the EV research field offering an unprecedented closer look to EV shape, composition and concentration. However, these technologies are very expensive and time-consuming, hindering their use in the clinical setting. FC is a well-established methodology but lack of sensitivity of conventional flow cytometers for sub-micron particle detection is an on-going limitation in $\mathrm{EV}$ research in terms of absolute quantification of EVs and reproducibility. Interestingly, Arraud et $\mathrm{al}^{39}$ presented a strategy to refine EV enumeration by using FC and fluorescence triggering wherein $\mathrm{EV}$ levels are generally higher as determined by fluorescence-based detection compared with light scatter-based detection. We observed that PMP levels from human plasmas were similar when using both light scatter or fluorescence triggering. These results align with a recent study showing superiority of light scatter detection of EVs over fluorescence triggering. ${ }^{36}$ Our values are also consistent with those from Brisson and colleagues ${ }^{39}$ when we enumerated 20,484 CD41a + ve PMPs per microlitre $( \pm 5,250)$ compared with 21,986 CD41a + ve PMPs per microlitre $( \pm 5,977)$. This cross-validation result confirms accurate and reliable quantification of CD41a + ve PMP subpopulations by nFC and light scatter detection only.

Superiority of nanoscale flow cytometers over conventional flow cytometers resides within significant improvements made for optical parameters and fluidic systems. For instance, unlike the majority of conventional flow cytometers using a high-powered blue laser (488 nm) to illuminate sub-micron particles, the A50Micro Plus utilizes a $405-\mathrm{nm}$ laser and a slow flow rate resulting in better sensitivity for particles below $200 \mathrm{~nm} .{ }^{37}$ To our knowledge, only Beckman Coulter also offers a flow cytometer (Cytoflex S) that relies on light scatter from EVs with a violet laser $(405 \mathrm{~nm})$. Calibration of FC instruments and standardization are required to attain inter-laboratory reproducibility and the validation of EVs as clinical biomarkers. In our study, the Apogee A50-Micro was calibrated for light-scatter resolution with silica-based calibration beads, whereas numerous studies erroneously encourage the use of latex/polystyrene beads. The latter exhibit high refractive indices that do not reflect those of EVs leading to under-estimation of EV concentrations and their sizes. In contrast, use of silica beads appears more relevant, exhibiting a RI closer to EVs.

$\mathrm{nFC}$, or in some cases, 'microflow' cytometry, will remain a cornerstone for $\mathrm{EV}$ analysis because the majority of experiments currently performed are of the immunophenotyping variety. This technology allows for high-throughput detection of specific MP populations and association with prognosis and outcome in various diseases. In our case, we performed two independent retrospective analyses in prostate cancer patients and leukaemia patients. Interestingly, we observed that PMP levels are not predictive of patient response to docetaxel in the prostate cancer study. In contrast, PMP levels tend to decrease in high-risk patients compared with low-risk patients. Both studies are limited by the small number of samples as well as discrepancy in sample preparation. However, it demonstrates that enumeration of PMPs can be standardized and performed when following a straightforward protocol. Proper calibration of nanoscale flow cytometers, standardization of sample processing and analytical conditions may allow for accurate determination of PMP concentrations in hundreds of patient samples within a week. Guidelines of this nature will have significant bearing as EVs like PMPs continue to have diagnostic and prognostic potential in the areas of oncology, haematology and infectious diseases.

\section{What is known about this topic?}

- Enumeration of platelet microparticles by conventional flow cytometry instrumentation is widely accepted but is unable to detect events smaller than $250 \mathrm{~nm}$ in diameter.

- Nanoscale/high-resolution flow cytometry is a new technology that is designed to analyse events that are between 100 and $1,000 \mathrm{~nm}$ in diameter and has been used to enumerate extracellular vesicles such as platelet microparticles.

\section{What does this paper add?}

- This paper provides key technical details regarding the detection limits of nanoscale flow cytometry and the optimal range of settings on this instrument for analysing extracellular vesicles such as platelet microparticles.

- Linearity in results, that is, the expected concentration obtained when the sample is serially diluted, is lost when the flow rate is too low, when the events of interest are close to the size of 'noise' and when the sample is analysed without any dilution.

- Analysis of platelet microparticle levels in the plasma samples of newly diagnosed prostate cancer patients that have responded to or failed chemotherapy revealed no significant difference. A decrease in platelet microparticle levels in low versus high RAI leukaemia patients was observed using this technique.

Authors' Contributions

J.G. and F.L. performed all experiments, designed all experiments and wrote the manuscript. Y.K., K.C.W., T.L., L.K. and F.L.L. performed experiments. C.M. and H.S. L. wrote the manuscript. N.E.K. and J.B. provided plasma samples and reviewed and edited the manuscript.

\section{Funding}

H.S.L. is supported by a Movember/Prostate Cancer Canada Rising Star Award (\#RS2012-008 and \#RS201656) and received operating grant support by Prostate 
Cancer Fight Foundation and Ontario Institute for Cancer Research (SPS 0613-03). Y.K. is supported by an Ontario Graduate Scholarship Award. K.C.W. is funded by a CIHR Postdoctoral Fellowship (140880). L.K. and F.L.L. are supported by the Natural Sciences and Engineering Research Council (NSERC) of Canada.

\section{Conflict of Interest}

O. Kenyon is the CEO of Apogee FlowSystems Inc. which manufactures the instrument (A50-Micro Plus) that is under evaluation in this article. The other authors report no conflict of interest.

\section{Acknowledgement}

The authors gratefully acknowledge the Nanofabrication Facility at Western University for SEM imaging.

\section{References}

1 Boulanger CM, Amabile N, Tedgui A. Circulating microparticles: a potential prognostic marker for atherosclerotic vascular disease. Hypertension 2006;48(02):180-186

2 Lynch SF, Ludlam CA. Plasma microparticles and vascular disorders. Br J Haematol 2007;137(01):36-48

3 George FD. Microparticles in vascular diseases. Thromb Res 2008; 122(Suppl 1):S55-S59

4 Barteneva NS, Fasler-Kan E, Bernimoulin M, et al. Circulating microparticles: square the circle. BMC Cell Biol 2013;14:23

5 Mause SF, Weber C. Microparticles: protagonists of a novel communication network for intercellular information exchange. Circ Res 2010;107(09):1047-1057

$6 \mathrm{Jy} \mathrm{W}$, Horstman LL, Jimenez JJ, et al. Measuring circulating cellderived microparticles. J Thromb Haemost 2004;2(10):1842-1851

7 Hargett LA, Bauer NN. On the origin of microparticles: from "platelet dust" to mediators of intercellular communication. Pulm Circ 2013;3(02):329-340

8 Wolf $\mathrm{P}$. The nature and significance of platelet products in human plasma. Br J Haematol 1967;13(03):269-288

9 Kornek M, Lynch M, Mehta SH, et al. Circulating microparticles as disease-specific biomarkers of severity of inflammation in patients with hepatitis $\mathrm{C}$ or nonalcoholic steatohepatitis. Gastroenterology 2012;143(02):448-458

10 Burger D, Schock S, Thompson CS, Montezano AC, Hakim AM, Touyz RM. Microparticles: biomarkers and beyond. Clin Sci (Lond) 2013;124(07):423-441

11 Xue S, Cai X, Li W, Zhang Z, Dong W, Hui G. Elevated plasma endothelial microparticles in Alzheimer's disease. Dement Geriatr Cogn Disord 2012;34(3-4):174-180

12 Diamant M, Tushuizen ME, Sturk A, Nieuwland R. Cellular microparticles: new players in the field of vascular disease? Eur J Clin Invest 2004;34(06):392-401

13 Matzdorff A, Kemkes-Matthes B, Pralle H. Microparticles and reticulated platelets in Wiskott-Aldrich syndrome patients. Br J Haematol 2000;109(03):673

14 Ando M, Iwata A, Ozeki Y, Tsuchiya K, Akiba T, Nihei H. Circulating platelet-derived microparticles with procoagulant activity may be a potential cause of thrombosis in uremic patients. Kidney Int 2002;62(05):1757-1763

15 Italiano JE Jr, Mairuhu ATA, Flaumenhaft R. Clinical relevance of microparticles from platelets and megakaryocytes. Curr Opin Hematol 2010;17(06):578-584

16 Burnouf T, Goubran HA, Chou M-L, Devos D, Radosevic M. Platelet microparticles: detection and assessment of their paradoxical functional roles in disease and regenerative medicine. Blood Rev 2014;28(04):155-166
17 Tseng C-C, Wang C-C, Chang H-C, et al. Levels of circulating microparticles in lung cancer patients and possible prognostic value. Dis Markers 2013;35(05):301-310

18 Platt M, Willmott GR, Lee GU. Resistive pulse sensing of analyteinduced multicomponent rod aggregation using tunable pores. Small 2012;8(15):2436-2444

19 Dragovic RA, Gardiner C, Brooks AS, et al. Sizing and phenotyping of cellular vesicles using Nanoparticle Tracking Analysis. Nanomedicine (Lond) 2011;7(06):780-788

20 Lawrie AS, Albanyan A, Cardigan RA, Mackie IJ, Harrison P. Microparticle sizing by dynamic light scattering in fresh-frozen plasma. Vox Sang 2009;96(03):206-212

21 Leong HS, Podor TJ, Manocha B, Lewis JD. Validation of flow cytometric detection of platelet microparticles and liposomes by atomic force microscopy. J Thromb Haemost 2011;9(12): 2466-2476

22 György B, Szabó TG, Turiák L, et al. Improved flow cytometric assessment reveals distinct microvesicle (cell-derived microparticle) signatures in joint diseases. PLoS One 2012;7(11):e49726

23 Abrams CS, Ellison N, Budzynski AZ, Shattil SJ. Direct detection of activated platelets and platelet-derived microparticles in humans. Blood 1990;75(01):128-138

24 van der Pol E, van Gemert MJC, Sturk A, Nieuwland R, van Leeuwen TG. Single vs. swarm detection of microparticles and exosomes by flow cytometry. J Thromb Haemost 2012;10(05): 919-930

25 Biggs CN, Siddiqui KM, Al-Zahrani AA, et al. Prostate extracellular vesicles in patient plasma as a liquid biopsy platform for prostate cancer using nanoscale flow cytometry. Oncotarget 2016;7(08): 8839-8849

26 Kibria G, Ramos EK, Lee KE, et al. A rapid, automated surface protein profiling of single circulating exosomes in human blood. Sci Rep 2016;6:36502

27 Chandler WL. Measurement of microvesicle levels in human blood using flow cytometry. Cytometry B Clin Cytom 2016;90 (04):326-336

28 Marcoux G, Duchez A-C, Cloutier N, Provost P, Nigrovic PA, Boilard E. Revealing the diversity of extracellular vesicles using high-dimensional flow cytometry analyses. Sci Rep 2016; 6:35928

29 van der Pol E, Coumans FAW, Grootemaat AE, et al. Particle size distribution of exosomes and microvesicles determined by transmission electron microscopy, flow cytometry, nanoparticle tracking analysis, and resistive pulse sensing. J Thromb Haemost 2014; 12(07):1182-1192

30 Montoro-García S, Shantsila E, Orenes-Piñero E, Lozano ML, Lip GY. An innovative flow cytometric approach for small-size platelet microparticles: influence of calcium. Thromb Haemost 2012; 108(02):373-383

31 van der Pol E, de Rond L, Coumans FAW, et al. Absolute sizing and label-free identification of extracellular vesicles by flow cytometry. Nanomedicine (Lond) 2018;14(03):801-810

32 Robert S, Poncelet P, Lacroix R, et al. Standardization of platelet-derived microparticle counting using calibrated beads and a Cytomics FC500 routine flow cytometer: a first step towards multicenter studies? J Thromb Haemost 2009;7(01): 190-197

33 Lacroix R, Judicone C, Poncelet $\mathrm{P}$, et al. Impact of pre-analytical parameters on the measurement of circulating microparticles: towards standardization of protocol. J Thromb Haemost 2012;10 (03):437-446

34 Coumans FAW, Brisson AR, Buzas EI, et al. Methodological guidelines to study extracellular vesicles. Circ Res 2017;120(10): 1632-1648

35 van der Pol E, Coumans FAW, Sturk A, Nieuwland R, van Leeuwen TG. Refractive index determination of nanoparticles in suspension using nanoparticle tracking analysis. Nano Lett 2014;14(11): 6195-6201 
36 de Rond L, van der Pol E, Hau CM, et al. Comparison of generic fluorescent markers for detection of extracellular vesicles by flow cytometry. Clin Chem 2018;64(04):680-689

37 van der Pol E, Sturk A, van Leeuwen T, Nieuwland R, Coumans F; ISTH-SSC-VB Working group. Standardization of extracellular vesicle measurements by flow cytometry through vesicle diameter approximation. J Thromb Haemost 2018;16(06):1236-1245

38 Stoner SA, Duggan E, Condello D, et al. High sensitivity flow cytometry of membrane vesicles. Cytometry A 2016;89(02): 196-206

39 Arraud N, Gounou C, Turpin D, Brisson AR. Fluorescence triggering: a general strategy for enumerating and phenotyping extracellular vesicles by flow cytometry. Cytometry A 2016;89(02): 184-195

40 Leong HS, Podor TJ, Manocha B, Lewis JD. Validation of flow cytometric detection of platelet microparticles and liposomes by atomic force microscopy. J Thromb Haemost 2011;9(12): 2466-2476

41 Gray WD, Mitchell AJ, Searles CD. An accurate, precise method for general labeling of extracellular vesicles. MethodsX 2015; 2:360-367
42 Bambace NM, Holmes CE. The platelet contribution to cancer progression. J Thromb Haemost 2011;9(02):237-239

43 Ogura H, Kawasaki T, Tanaka H, et al. Activated platelets enhance microparticle formation and platelet-leukocyte interaction in severe trauma and sepsis. J Trauma 2001;50(05):801-809

44 van Doormaal F, Kleinjan A, Berckmans RJ, et al. Coagulation activation and microparticle-associated coagulant activity in cancer patients. An exploratory prospective study. Thromb Haemost 2012;108(01):160-165

45 Helley D, Banu E, Bouziane A, et al. Platelet microparticles: a potential predictive factor of survival in hormone-refractory prostate cancer patients treated with docetaxel-based chemotherapy. Eur Urol 2009;56(03):479-484

46 Cointe S, Judicone C, Robert S, et al. Standardization of microparticle enumeration across different flow cytometry platforms: results of a multicenter collaborative workshop. J Thromb Haemost 2017;15(01):187-193

47 Andersen MN, Al-Karradi SNH, Kragstrup TW, Hokland M. Elimination of erroneous results in flow cytometry caused by antibody binding to FC receptors on human monocytes and macrophages. Cytometry A 2016;89(11):1001-1009 